Trauma Berufskrankh 2014 · 16:5 DOI 10.1007/s10039-014-2063-9

Online publiziert: 22. Februar 2014

c) Springer-Verlag Berlin Heidelberg 2014

R. Hoffmann · K. Schmidt-Horlohé

Berufsgenossenschaftliche Unfallklinik Frankfurt am Main

\title{
Frakturen und Sehnenverletzungen des Schultergürtels
}

beurteilung inzwischen Standard. In diesem Kontext stellt sich die Frage, ob sich diese zunehmenden diagnostischen Möglichkeiten auch in entsprechende Therapieerfolge umsetzen lassen.

In der Therapie der Schultergürtelverletzungen kam es in den vergangenen Jahren zu vielen Weiterentwicklungen. Es wird mehr erkannt, neu klassifiziert - und mehr operiert, dies jedoch nicht immer auf bereits evidenzgesicherter Basis oder mit nachgewiesen besseren klinischen Ergebnissen. Neue winkelstabile Plattensysteme konnten nicht immer die in sie gesetzten Erwartungen erfüllen. Auch die Schulterendoprothetik nach einem Trauma bleibt in ihrer Durchführung und Prognose schwierig. Die Indikation zur inversen Endoprothese als letzter Verteidigungslinie ist sorgsam und kritisch im Einzelfall abzuwägen und sollte - wie ihre Implantation selbst - dem hierin Erfahrenen vorbehalten bleiben. Minimalinvasive Techniken und arthroskopisch unterstützte oder durchgeführte Eingriffe erfordern besondere Expertise und Spezialistentum. Sie sollten nicht unkritisch und ohne wissenschaftlich sauber nachgewiesene Evidenz zum neuen Standard erklärt werden. Somit bedarf die Versorgung von Schulterverletzungen noch der weiteren Optimierung - v. a. gut geplanter prospektiv-randomisierter klinischer Studien sowie der Erweiterung der aufkommenden Register- und Versorgungsforschung.

Wir bedanken uns ausdrücklich bei den Autoren als ausgewiesene Experten für ihre Beiträge zu diesem Leitthema.
Die detaillierten und wissenschaftlich begründeten Artikel ermöglichen einen Überblick über den aktuellen Stand von Ätiologie, Diagnostik, Klassifikation und Therapie von Frakturen und Sehnenverletzungen des Schultergürtels.

Frankfurt, Februar 2014

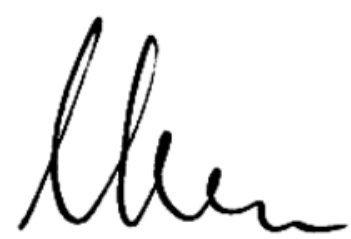

\section{R. Hoffmann}

und

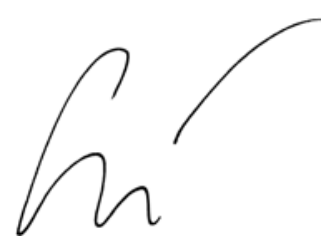

K. Schmidt-Horlohé

\section{Korrespondenzadresse}

Prof. Dr. R. Hoffmann

Berufsgenossenschaftliche Unfallklinik

Frankfurt am Main,

Friedberger Landstraße 430,

60389 Frankfurt am Main

aerztlicher.direktor@bgu-frankfurt.de

Interessenkonflikt. R. Hoffmann und K. SchmidtHorlohé geben an, dass kein Interessenkonflikt besteht. 